\title{
Riqueza y abundancia de mariposas diurnas, escarabajos coprófagos y plantas en cultivos orgánicos y convencionales de tres regiones de Costa Rica
}

\author{
Natalie V. Sánchez ${ }^{1}$, Luis Esteban Vargas-Castro ${ }^{2}$, Angie Sánchez ${ }^{3}$ y Manuel Amador ${ }^{4}$ \\ 1. Escuela de Biología, Universidad de Costa Rica, Sede Occidente, Alajuela, Costa Rica; natingui@gmail.com \\ 2. Department of Biology, University of Miami, Coral Gables, FL 33146, USA; luissum@gmail.com \\ 3. Unidad de Biología del Departamento de Gestión Ambiental del Proyecto Hidroeléctrico Reventazón, Instituto Costarricense de Electricidad, \\ San José, Costa Rica; asanchezn@ice.go.cr \\ 4. Corporación Educativa para el Desarrollo Costarricense (CEDECO); manuel@cedeco.or.cr
}

Recibido 28-II-2013 Corregido 29-IV-2013 Aceptado 7-V-2013

\begin{abstract}
Butterflies, dung beetles, and plant species richness and abundance in organic and conventional crops in three regions of Costa Rica. One of the main causes of biodiversity loss is related to agriculture increase and the use of chemicals. Organic farming has been proposed as a solution to biodiversity loss, for that reason we evaluated the species richness, abundance and composition of diurnal butterflies, dung beetles, herbs and trees in three different agroecosystems during 2009-2010 in Costa Rica. Organic and non-organic coffee shade (San José), vegetables (Alajuela) and Cocoa-banana farms (Talamanca) were selected to make comparisons of these biological groups. In each farm we established three transects to capture butterflies, 20 pitfall traps to capture beetles, two plots to count trees and two sub-plots to count herbs. The Cocoa-banana organic crops had higher species richness of butterflies and higher mean abundance than coffee shade and cocoa-banana crops. We detected a greatest abundance of beetles in organic farms of shade coffee and cocoa-banana. In the vegetable and cocoa-banana crops there was more abundance of herbs. Organic cocoa-banana had more trees species and they are more abundant than in conventional crops. Butterflies, herbs and trees were less similar in species composition between organic and non-organic crops; however beetles were more similar between systems. Organic shade coffee and cocoa-banana are providing a more diverse habitat and resources to butterflies than vegetables farms. The more complex plant structure, the shaded habitats and mammals present in shade coffee and cocoa-banana organic farms favor beetle abundance. In addition, beetles could be adapted to altered ecosystems. Organic farms can play an important role in butterfly and beetles conservation in countryside areas, and its benefits are influenced by forest presence and the manner in which farmers manage their land.
\end{abstract}

\section{KEY WORDS}

Dung beetles, butterflies, organic agriculture, cocoa plantation, shadegrown coffee

\section{RESUMEN}

Una de las principales causas de pérdida de biodiversidad es la expansión de la frontera agrícola, así como el uso de químicos. La agricultura orgánica se propone como una alternativa de producción asociada a la conservación de biodiversidad, en este sentido evaluamos la riqueza, abundancia y composición de tres grupos taxonómicos (mariposas diurnas, escarabajos coprófagos y plantas) presentes en tres regiones de Costa Rica en 2009 - 2010, en tres cultivos orgánicos y tres convencionales de café (San José), hortalizas (Alajuela) y cacao y plátano (Talamanca). En cada tipo de cultivo capturamos mariposas, colocamos 20 trampas de caída para capturar escarabajos, establecimos dos parcelas para registrar árboles y hierbas presentes. Los cultivos orgánicos de cacao-banano tuvieron mayor riqueza de mariposas. En promedio las mariposas y escarabajos fueron más abundantes en café y cacao orgánico. En los cultivos orgánicos de hortalizas y cacao-banano hubo mayor abundancia de hierbas. Varios factores favorecen la presencia de estos grupos biológicos en cultivos orgánicos, los cuales proporcionan un hábitat más heterogéneo que los convencionales, evitando el uso de herbicidas y plaguicidas. Además, los cultivos orgánicos pueden desempeñar un papel importante como zonas de paso entre bosques para estos grupos, por tanto el bosque influye en su presencia en los cultivos y la persistencia a largo plazo en la forma en que los agricultores manejan sus tierras.

\section{PALABRAS CLAVE}

Escarabajos coprófagos, mariposas, agricultura orgánica, plantación de cacao, café con sombra 
La expansión de la frontera agrícola, es considerada una de las causas más importantes de pérdida de biodiversidad (Swift et al., 1996). Esta expansión justificada por el aumento en la demanda de alimentos, ha provocado una intensificación del área de monocultivos dependientes de grandes cantidades de fertilizantes, herbicidas y pesticidas, generando impacto negativo sobre el ambiente (Matson, Parton, Power \& Swift, 1997). Ante esta situación, se ha vuelto una necesidad el desarrollo de métodos de gestión agrícola que permitan armonizar la producción agraria con el desarrollo rural y la conservación de los recursos naturales (Sans, 2007). La agricultura orgánica es una alternativa de conservación ya que se busca el menor uso de insumos externos, favoreciendo la producción interna y la conservación de la biodiversidad (Hald, 1999; Castro \& Amador, 2007).

El potencial de los sistemas agrícolas para la preservación de la biodiversidad, es reconocido al ser adecuadamente manejados (Rivera \& Ambrecht, 2005). Algunos estudios han evaluado el aporte de la agricultura orgánica en la conservación de la biodiversidad y de grupos biológicos como aves y artrópodos que pueden ser indicadores de la sostenibilidad ambiental de las prácticas agrícolas realizadas en los cultivos (Florian, 2005; Guillen, SotoAdames, \& Springer, 2006; George, 2006). Por ejemplo, los insectos cumplen papeles ecológicos importantes así como indicadores de la salud del agroecosistema, debido a que su ciclo de vida está fuertemente influenciado por la estructura y calidad del hábitat (Jeanneret, Schüpbach, Pfiffner \& Walter, 2003; Perfecto, Mas, Dietsch \& Vandermeer, 2003; Pérez, 2008). Dentro de este grupo, las mariposas cumplen roles ecológicos importantes que tienen impactos en los cultivos como herbivoría y polinización. Además, la respuesta que este grupo presenta a los procesos de disturbio o alteración es rápida (Finegan, Hayes, Delgado \& Gretzinger, 2004), y pueden ser muy sensibles a cambios en la cobertura vegetal debido a su dependencia a las plantas hospederas y a las variaciones microclimáticas (Thomas, 1991; Brown \& Hutchings, 1997). Por otra parte, los escarabajos coprófagos poseen una gran importancia ecológica como recicladores de nutrientes, los cuales renuevan y fertilizan constantemente el suelo actuando como indicadores de la calidad de hábitat (Morón, 2004; Hansky \& Cambefort, 1991). Así mismo, participan en el enterramiento de semillas en el suelo, cumpliendo el papel de dispersores secundarios (Vulinec, 2000), y son indicadores indirectos de la presencia de vertebrados (Klein, 1989; Hernández et al., 2003).

Otro grupo indicador de la diversidad presente en cultivos es la vegetación acompañante, ya que están íntimamente asociadas a las actividades del agro y es un claro reflejo de los efectos del uso de la tierra y de la sostenibilidad del sistema de producción (Becker, 1995). En sistemas de producción orgánicos la presencia de vegetación brinda productos alternativos que favorecen los ingresos del productor y al mismo tiempo mejora la calidad de hábitat para algunos grupos biológicos como aves, murciélagos y artrópodos (Fuller et al., 2005), mientras que en sistemas de producción intensiva es considerada competencia para el cultivo. Teniendo en cuenta la importancia ecológica de los grupos previamente descritos en la conservación de la biodiversidad, el objetivo de esta investigación fue comparar la riqueza y abundancia de mariposas diurnas, escarabajos coprófagos y vegetación acompañante, así como la composición de especies en fincas con diferentes cultivos orgánicos con respecto a fincas de producción convencional en tres zonas de Costa Rica.

\section{METODOLOGÍA}

El muestreo de los grupos biológicos se realizó en tres fincas de producción orgánica, tres fincas de producción convencional y un área de bosque en tres localidades de Costa Rica: Caraigres, San José $\left(9^{\circ} 49^{\prime} 11^{\prime \prime} \mathrm{N}-84^{\circ} 07^{\prime} 57^{\prime \prime} \mathrm{W}\right)$, Alfaro Ruiz, Alajuela $\left(10^{\circ} 14^{\prime} 69^{\prime \prime} \mathrm{N}-84^{\circ} 22^{\prime} 80^{\prime \prime} \mathrm{W}\right)$ y Shiroles, Talamanca, Limón $\left(9^{\circ} 36^{\prime} 29^{\prime \prime} \mathrm{N}-82^{\circ} 52^{\prime} 03^{\prime \prime} \mathrm{W}\right)$. En Caraigres y Alfaro Ruíz, el muestreo se realizó entre los meses de marzo a mayo del 2009 y en Talamanca de noviembre a diciembre del 2010. Las fincas orgánicas y convencionales en Caraigres son de café, en Alfaro Ruíz de hortalizas (lechuga, culantro, cebollino, brócoli) y en Talamanca los cultivos orgánicos son una combinación de cacao-banano y los convencionales, plantaciones de plátano. Caraigres pertenece a las zonas de vida bosque muy húmedo premontano, con una precipitación promedio anual de $2500-3000 \mathrm{~mm}$ y una temperatura media $23,5^{\circ} \mathrm{C}$ (Tosi, 1969). El cantón de Alfaro Ruíz se encuentra en la zona de vida bosque muy húmedo montano bajo, la precipitación promedio anual es $2500 \mathrm{~mm}$ y la temperatura promedio $16,5^{\circ} \mathrm{C}$. La región de Shiroles, Talamanca presenta una precipitación promedio anual entre $1900-2700 \mathrm{~mm}$ y una temperatura que varía de $22-27^{\circ} \mathrm{C}$. Esta región se caracteriza por la presencia de bosque tropical húmedo y bosque premontano húmedo (Tosi, 1969).

Muestreo de insectos y vegetación: En cada finca orgánica y convencional (tipo de manejo) se establecieron tres transectos de $80 \times 6 \mathrm{~m}$ para el muestreo de mariposas diurnas, el cual se realizó entre las 0800-1100hr. Durante este periodo, se colectaron, identificaron y se contabilizaron el número de individuos por especie. Cada transecto fue recorrido por dos personas durante una hora por día, durante tres días consecutivos y la captura se realizó con redes entomológicas. Para la identificación de las especies de mariposas se utilizó la guía "Butterflies of Costa Rica, 
and their natural history", volúmenes I y II (DeVries, 1987). Los especímenes que no pudieron ser identificados fueron llevados al Museo Nacional.

La colecta de escarabajos se realizó con trampas de caída "pitfall". Se colocaron 20 trampas por tipo de manejo, distribuidas cada $20 \mathrm{~m}$ en los transectos ya establecidos para el muestreo de mariposas. Las trampas consistieron en recipientes plásticos enterrados a nivel del suelo, los cuales tenían en su interior agua con jabón sin olor. Sobre el vaso se colocó una malla de $2,5 \times 1,25 \mathrm{~cm}$, para colocar el cebo de estiércol de cerdo (Finegan et al., 2004). Las trampas fueron cebadas y revisadas diariamente durante tres días, registrando las especies y el número de individuos por especie capturadas. Los especímenes colectados se conservaron en envases plásticos con alcohol al 70\%. Las especies se identificaron en el Instituto Nacional de Biodiversidad (INBio).

Finalmente, en cada finca se establecieron dos parcelas cuadrangulares de $2 \times 2 \mathrm{~m}$, donde se registró la riqueza y abundancia de plantas herbáceas y arbustivas menores a $75 \mathrm{~cm}$ de altura. En las fincas de Caraigres y Talamanca se establecieron dos parcelas cuadrangulares de 10x10m para el muestreo de especies arbóreas por tipo de manejo. En cada parcela se registraron todas aquellas especies arbóreas con una altura mayor a 1,5m. Las especies que no pudieron ser identificadas en el campo fueron llevadas al Herbario de la Escuela de Ciencias Ambientales de la Universidad Nacional para su posterior identificación.

Análisis de datos: Se realizó un análisis de varianza para determinar si existían diferencias entre los sistemas orgánicos, convencionales por sitio en término de riqueza de especies y abundancia de escarabajos, mariposas y vegetación acompañante. Antes de realizar los análisis de varianza, se revisó la normalidad de los datos; aquellos sin una distribución normal fueron analizados mediante la prueba no paramétrica de Kruskal-Wallis. Todas las pruebas estadísticas se realizaron con el programa $R$ versión 2.14.1 (R Development Core Team, Vienna, Austria). La composición de especies encontradas en cada tipo de cultivo se comparó usando el coeficiente de similitud de Jaccard (Real \& Vargas, 1996).

\section{RESULTADOS}

En las fincas de café en Caraigres se colectaron un total de 14 especies de escarabajos, 12 especies en orgánico, 10 en convencional y 4 en bosque. Los géneros con mayor número de individuos fueron Onthophagus y Eurysternus (Cuadro 1). No hubo diferencias en la riqueza promedio de escarabajos ( $F=3,524 ; D f=16 ; p=0,0788)$, pero la abundancia fue mayor en cultivos orgánicos que en convencionales ( $F=6,001 ; D f=16 ; p=0,0262)$ (Fig. 1A). En Alfaro Ruíz se colectaron 5 especies de escarabajos, con 4 especies para cada tipo de cultivo y bosque. La abundancia de escarabajos fue similar entre cultivos convencionales y cultivos orgánicos ( $F=2,18, D f=16 ; p=0,159)$. En Talamanca, se identificaron 16 especies de escarabajos, 14 en orgánico, cuatro en convencional y nueve en bosque. La especie detectada con mayor número de individuos fue Onthophagus acuminatus. La riqueza de especies fue similar entre los tipos de cultivo $(H=3,23, p=0,07)$, pero la abundancia de escarabajos fue mayor en orgánico $(F=35,41 ; D f=4 ; p=0,004)$ (Fig. 1B). En las tres localidades, el género Onthophagus tuvo la mayor riqueza y abundancia de especies tanto en los cultivos orgánicos como en los convencionales (Cuadro 1).
A

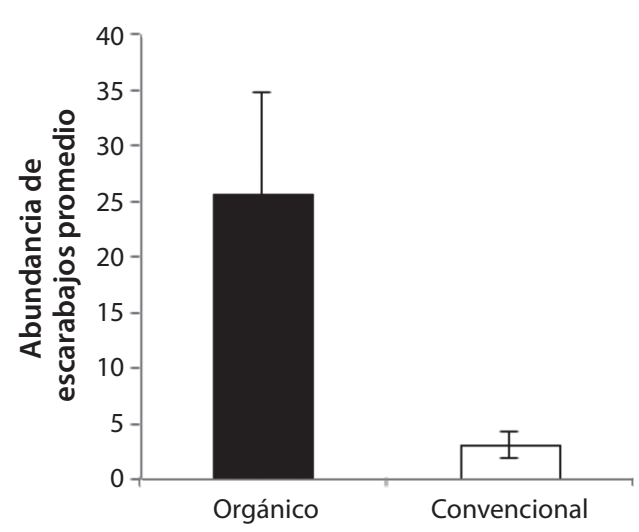

B

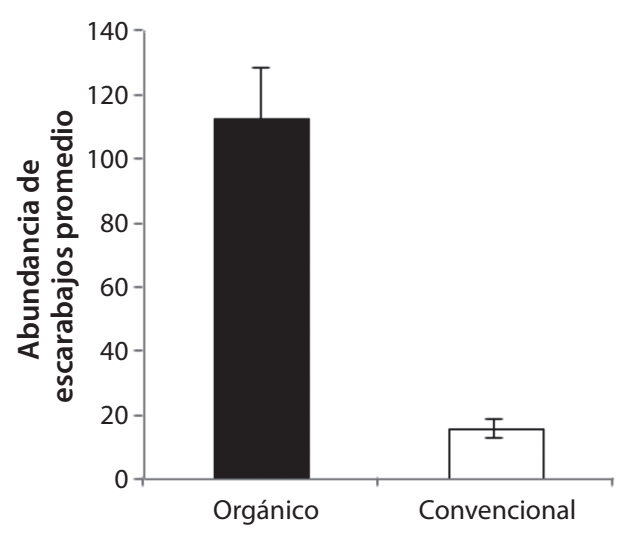

FIG. 1. (A) Abundancia de escarabajos coprófagos promedio ( $\pm \mathrm{EE}$ ) registrados en fincas orgánicas y convencionales de café en el área de Caraigres (B) y de cacao (orgánico) plátano (convencional) en Talamanca, Costa Rica, $2009-2010$. 
CUADRO 1

Mariposas y escarabajos más comunes en cultivos orgánicos (Org), no orgánicos (NoOrg) y bosques de tres regiones de Costa Rica, 2009-2010.

\begin{tabular}{|c|c|c|c|c|}
\hline & Especie & Org & NoOrg & Bosque \\
\hline \multicolumn{5}{|l|}{ Caraigres } \\
\hline \multirow[t]{5}{*}{ Escarabajos } & Canthon aequinoctealis & 49 & 0 & 0 \\
\hline & Canthon caelius & 36 & 0 & 0 \\
\hline & Eurysternus mexicanus & 29 & 3 & 1 \\
\hline & Eurysternus plebejus & 39 & 4 & 0 \\
\hline & Onthophagus landolti & 62 & 7 & 7 \\
\hline \multirow[t]{10}{*}{ Mariposas } & Anartia fatima & 36 & 2 & 0 \\
\hline & Cissia hermes & 56 & 11 & 6 \\
\hline & Cissia hescione & 55 & 14 & 0 \\
\hline & Cissia libye & 22 & 4 & 0 \\
\hline & Cissia retana & 27 & 0 & 5 \\
\hline & Cissia usitata & 34 & 2 & 1 \\
\hline & Eurema daira & 66 & 1 & 0 \\
\hline & Heliconius charitonius & 22 & 2 & 0 \\
\hline & Heliconius erato petiverana & 52 & 6 & 10 \\
\hline & Pareuptychia metaleuca & 21 & 0 & 0 \\
\hline \multicolumn{5}{|l|}{ Talamanca } \\
\hline \multirow[t]{5}{*}{ Escarabajos } & Onthophagus acuminatus & 128 & 32 & 50 \\
\hline & Onthophagus limonensis & 97 & 0 & 8 \\
\hline & Onthophagus batesi & 37 & 11 & 5 \\
\hline & Canthon meridionalis & 22 & 0 & 22 \\
\hline & Eurysternus mexicanus & 11 & 3 & 0 \\
\hline \multirow[t]{4}{*}{ Mariposas } & Anartia fatima fatima & 21 & 0 & 8 \\
\hline & Cissia confusa & 16 & 4 & 6 \\
\hline & Hermeuptychia harmonia & 16 & 20 & 4 \\
\hline & Hermeuptychia Hermes & 33 & 25 & 0 \\
\hline \multicolumn{5}{|l|}{ Alfaro Ruíz } \\
\hline \multirow[t]{5}{*}{ Escarabajos } & Onthophagus incensus & 1192 & 2341 & 121 \\
\hline & Canthon meridionalis & 0 & 0 & 199 \\
\hline & Copris lugubris & 19 & 30 & 7 \\
\hline & Onthophagus anthracinus & 139 & 220 & 0 \\
\hline & Onthophagus cyanellus & 336 & 518 & 371 \\
\hline \multirow[t]{7}{*}{ Mariposas } & Anthanassa ardys & 33 & 28 & 1 \\
\hline & Copaeodes sp. & 14 & 17 & 0 \\
\hline & Dione moneta poeyii & 39 & 28 & 8 \\
\hline & Echinargus isola & 13 & 38 & 0 \\
\hline & Greta anette & 4 & 0 & 19 \\
\hline & Leptophobia aripa & 113 & 89 & 23 \\
\hline & Vanessa virginiensis & 26 & 28 & 0 \\
\hline
\end{tabular}


En cuanto a la riqueza de mariposas en Caraigres se registró un total de 102 especies, 54 en orgánico, 57 en convencional y 24 en bosque, distribuidas en número similar entre los dos tipos de manejo ( $F=4,404 ; D f=16 ; p=0,0521)$. Se registró mayor abundancia de individuos en cultivo orgánico que en convencional ( $F=18,23 ; \mathrm{Df}=16 ; \mathrm{p}=0,00058)$ (Fig. 2). Cissia y Eurema fueron los géneros de mariposas más comunes (Cuadro 1). En Alfaro Ruíz, se capturaron 30 especies de mariposas, con una riqueza de especies similar entre sistemas orgánicos (21 spp) y convencionales (16 spp) $(F=0,315 ; D f=16 ; p=0,582)$. Igualmente, la abundancia de mariposas fue similar tanto en cultivos orgánicos como cultivos convencionales ( $F=0,053 ; D f=16 ; p=0,82)$ (Fig. 2). Para Talamanca, se identificaron 32 especies de mariposas, 26 en orgánico, 13 en convencional y 14 en bosque. Las especies más comunes y que además estuvieron presentes en los dos tipos de manejo fueron Hermeuptychia harmonia y $\mathrm{H}$. hermes, seguida por mariposas de la especie Anartia fatima y Cissia confusa comunes para cultivos orgánicos (Cuadro 1). En promedio los cultivos orgánicos de cacao-banano tienen mayor riqueza de especies de mariposas diurnas $(F=$ 44,$76 ; \mathrm{Df}=16 ; \mathrm{p}<0,001$ ) (Fig. 3) y mayor abundancia promedio $(F=22,69 ; D f=16 ; p=0,0002)$ que los cultivos de plátano convencional (Fig. 2).

En Caraigres, se identificaron 47 árboles utilizados como sombra del café, 23 en orgánico, 21 en convencional, distribuidas similarmente en términos de riqueza ( $F=$ $1,767 ; \mathrm{Df}=36 ; \mathrm{p}=0,193)$ y de abundancia de individuos entre los dos tipos de cultivo $(F=0,86 ; D f=34 ; p=0,36)$. El género Musa fue el más utilizado como sombra dentro de los sistemas orgánico y convencional. En el caso de hierbas

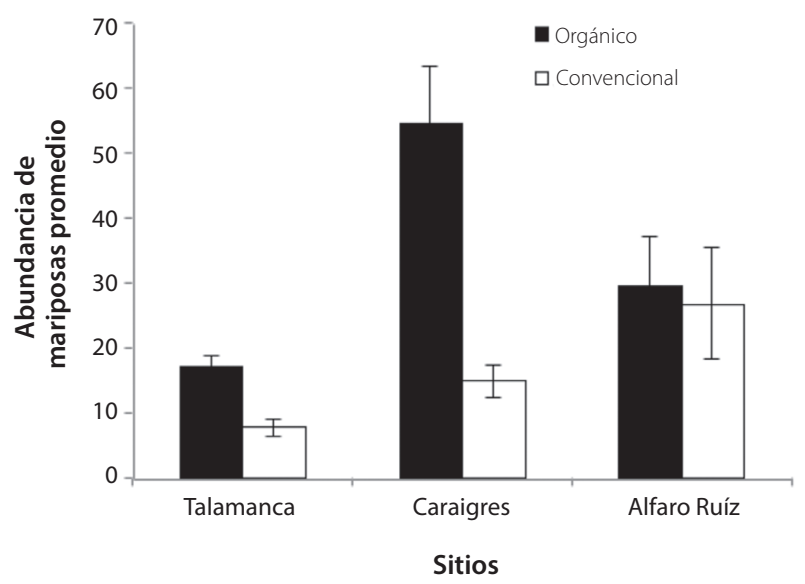

FIG. 2. Abundancia de mariposas promedio ( $\pm \mathrm{EE}$ ) registradas en fincas orgánicas y convencionales de café en Caraigres, hortalizas en Alfaro Ruiz y cacao-banano en Talamanca, Costa Rica, 2009-2010. acompañantes, se registró un total de 84 especies, 48 en orgánicos y 39 en convencionales. El número de especies promedio y la abundancia de individuos fueron similares entre los dos sistemas de producción (Cuadro 2). En Alfaro Ruiz, registramos un total de 20 especies de herbáceas, 19 en orgánico y 13 en convencional. Para este grupo sí fue posible detectar una diferencia significativa en la riqueza y abundancia de especies herbáceas entre los sistemas productivos evaluados, siendo mayor en cultivos orgánicos que en cultivos convencionales (Cuadro 2). Por otra parte, para Talamanca, identificamos 19 especies de árboles, en los cultivos de cacao-banano se identificaron 15 especies y en los convencionales de plátano solamente 5 especies. En los cultivos de cacao-banano hay un número similar de especies de árboles que en los cultivos convencionales $(F=4,865 ; D f=10 ; p=0,0519)$ (Fig. $4 A)$, pero hubo mayor abundancia promedio por especie que en los cultivos convencionales ( $F=10,29 ; D f=10 ; p=0,00936)$ (Fig. 4B). En lo que respecta a las hierbas acompañantes, se detectaron 22 especies, 17 en los cultivos de cacao-banano y 10 en cultivos de plátano convencional. La riqueza promedio de hierbas acompañantes fue mayor en los cultivos orgánicos que en los cultivos convencionales de plátano $(\mathrm{H}=$ 4,68; $p=0,03057)$, así como la abundancia promedio $(H=$ 8,31; $p=0,003948$ ) (Cuadro 2).

\section{Composición de especies}

La composición de especies en los cultivos de café fue diferente entre cultivos orgánicos y convencionales para todos los grupos evaluados. El grupo que mostró mayor

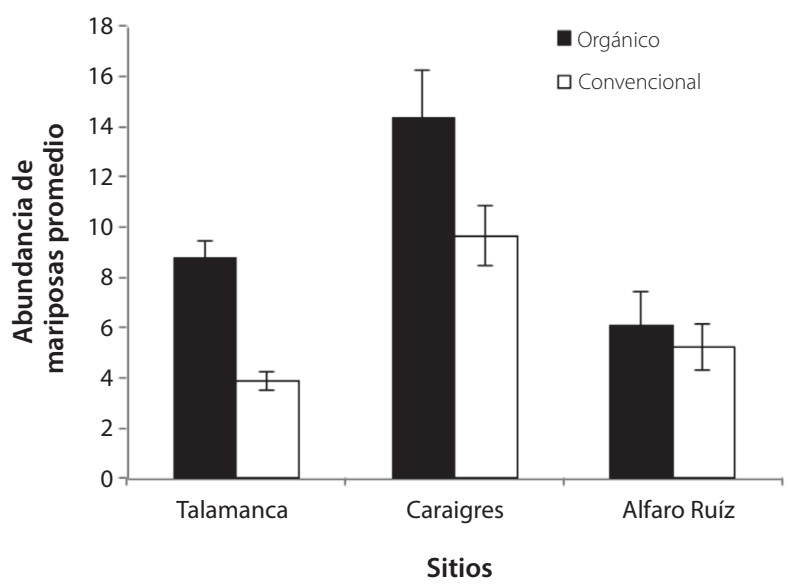

FIG. 3. Riqueza de mariposas promedio ( $\pm \mathrm{EE}$ ) registradas en fincas orgánicas y convencionales de café (Caraigres) hortalizas (Alfaro Ruiz) y cacao-banano (Talamanca), Costa Rica, 2009-2010. 


\section{CUADRO 2}

Comparación de la riqueza y abundancia promedio de hierbas acompañantes entre cultivos orgánicos y convencionales en tres regiones de Costa Rica, 2009-2010.

\begin{tabular}{lcccccc}
\hline & \multicolumn{1}{c}{ Riqueza } & \multicolumn{3}{c}{ Abundancia } \\
\hline \multicolumn{1}{c}{ Sitio } & Orgánico & Convencional & Valor $\mathbf{p}$ & Orgánico & Convencional & Valor $\mathbf{p}$ \\
\hline $\begin{array}{l}\text { Caraigres (café) } \\
\begin{array}{l}\text { Alfaro Ruíz } \\
\text { (hortalizas) }\end{array}\end{array}$ & 4,4 & 3,4 & 0,17 & 17,9 & 15,7 & 0,71 \\
$\begin{array}{l}\text { Talamanca } \\
\text { (cacao-platano) }\end{array}$ & 8,3 & 3,6 & $0,002^{*}$ & 237,7 & 94,55 & $0,02^{*}$ \\
* & 6,8 & 4,0 & $0,004^{*}$ & 888,7 & 325,7 & $0,03^{*}$ \\
\end{tabular}

A

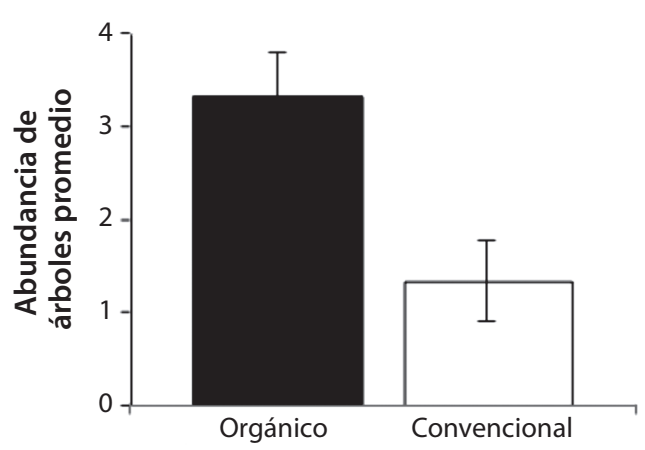

B

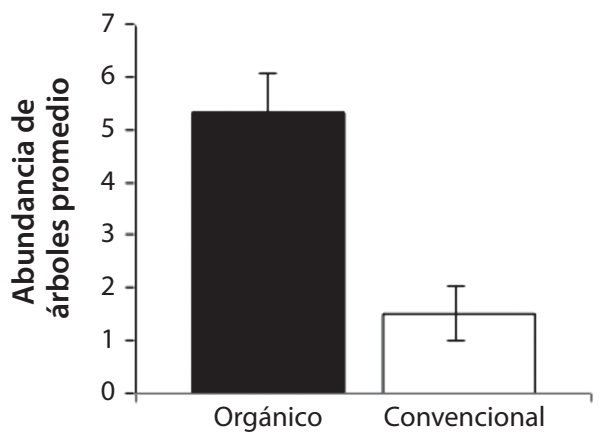

FIG. 4. (A) Riqueza y (B) abundancia de árboles (> 1,5 m de altura) ( \pm EE) en fincas orgánicas de cacao-banano y plantaciones de plátano convencional. Diciembre 2010, Talamanca, Costa Rica.

similitud de especies fueron los escarabajos quienes comparten el $57 \%$ de las especies. Cuatro especies de escarabajos únicamente fueron registradas en cultivos orgánicos: Canthidium ardens, Canthon aequinoctealis, C. caelius y Copris lugubris, mientras que la especie Onthophagus acuminatus solamente se registró en convencionales. Sólo el $25 \%$ de las mariposas, el $29 \%$ de la vegetación para sombra y el $23 \%$ de hierbas acompañantes estuvieron presentes en ambos cultivos (Cuadro 3). Se detectaron 19 mariposas únicas para cultivos orgánicos y 33 especies únicas para cultivos convencionales (Cuadro 4), siendo la más común Megeuptychia antinoe para orgánico y Calephelis sodalisi en sistemas convencionales.

En Caraigres los árboles más comunes fueron laurel (Cordia alliodora), cocobolo (Dalbergia retusa), guanacaste (Enterolobium cyclocarpum) y uruca (Trichilia americana). Las especies únicas en cultivos convencionales fueron: gravillea (Grevillea robusta), teca (Tecoma stans), guaba (Inga spectabilis) y cirrí colorado (Mauria heterophylla). En Alfaro Ruíz un $37 \%$ de las especies de mariposas fueron compartidas entre tratamientos. Por otro lado, los escarabajos compartieron la totalidad de las especies registradas en ambos sistemas productivos (Cuadro 3). La evaluación de la vegetación evidenció que el $60 \%$ de hierbas acompañantes estuvieron presentes tanto en cultivos de hortalizas orgánicos como en convencionales, un $37 \%$ de las especies fueron registrados únicamente en cultivos orgánicos y un $5 \%$ fueron exclusivas a cultivos convencionales (Cuadro 3). De las especies de mariposas, 11 especies fueron registradas únicamente en cultivos orgánicos, mientras que en cultivos convencionales se registraron cinco especies (Cuadro 4). Para los escarabajos, a pesar que en los sistemas productivos evaluados se compartió la totalidad de las especies registradas, en el bosque se registró una especie única, Canthon meridionales, y no se registró la presencia de la especie Onthophagus anthracinus. Entre las hierbas más comunes que se registraron exclusivamente en fincas orgánicas se encuentran: moriseco (Bidens pilosa), cinquitos (Drymaria cordata) y escobilla (Elephantopus mollis). Por otro lado, en los cultivos convencionales Cardamine bonariensis fue única para estos sitios. 
En Talamanca los cultivos orgánicos de cacao-banano compartieron $36 \%$ de las especies de mariposas con los cultivos convencionales (Cuadro 3) y compartieron más especies con el bosque. Solamente 19 especies se detectaron en cultivos orgánicos (Cuadro 4). En los cultivos convencionales una especie fue específica para este sitio, $\mathrm{Ha}$ des noctula. Las especies compartidas tanto en el bosque como en los dos tipos de cultivos fueron Cissia confusa, Hermeuptychia harmonia, Magneuptychia libye y Mechanitis polymnia isthmia (Cuadro 4). Para el grupo de los escarabajos, el cultivo orgánico de cacao-banano fue más similar al bosque que el cultivo de plátano convencional. Las especies compartidas entre los tres tratamientos fueron las especies Onthophagus acuminatus y Onthophagus batesi. Interesantemente los dos tipos de cultivos son más similares entre sí que al bosque en cuanto a la composición de especies, esto quiere decir que el bosque tiene algunas especies únicas que lo hacen diferente a los cultivos.

\section{DISCUSIÓN}

La mayor abundancia de mariposas en los cultivos de café y cacao-banano se puede explicar por la presencia de más número de especies de hierbas y árboles en estos cultivos que están proveyendo alimento, tanto para los adultos como para las larvas (Gallina, Mandujano, \& Gonzalez-Romero, 1996; Moguel \& Toledo, 1999; Schroth et al., 2004). A esto se le une la ausencia en el uso de herbicidas o agroquímicos, permitiendo mayor variedad de cobertura vegetal (Feber, Firbank, Johnson \& Macdonald, 1997; Hole et al., 2005). Por otra parte, el café y el cacao pueden crecer bajo la sombra de otros árboles y en compañía de otros cultivos permitiendo albergar más riqueza de especies que otros (Guiracocha, Harvey, Somarriba, Krauss \& Carrillo, 2001).

En las fincas de café se registró una importante cantidad de especies de mariposas típicas de áreas abiertas. Coincidiendo con Horner-Devine, Daily, Ehrlich y Boggs (2003) que determinaron que las especies de mariposas no frugívoras son más comunes de encontrar en áreas abiertas como las plantaciones de café que en hábitats de bosque. En Talamanca, los cultivos orgánicos de cacao, comúnmente son sembrados junto al banano y el área dedicada para su producción se combina con especies frutales como Carica papaya, Citrus limettioides y Syzygium malaccense lo que contribuye a aumentar la heterogeneidad en el cultivo para beneficio de las mariposas. En contraste, los cultivos convencionales de plátano son hábitats pobres en cuanto a riqueza de especies de árboles o hierbas, por tanto, pocas especies de mariposas diurnas

CUADRO 3

Índice de Similitud de Jaccard para escarabajos coprófagos, mariposas, árboles y hierbas acompañantes de cultivos orgánico y convencional, en tres regiones de Costa Rica, 2009-2010.

\begin{tabular}{llcccc}
\hline \multicolumn{1}{c}{ Sitios } & $\begin{array}{c}\text { Grupo } \\
\text { biológico }\end{array}$ & $\begin{array}{c}\text { Orgánico } \\
\text { (spp. únicas) }\end{array}$ & $\begin{array}{c}\text { Convencional } \\
\text { (spp. únicas) }\end{array}$ & $\begin{array}{c}\text { Spp. } \\
\text { compartidas }\end{array}$ & $\begin{array}{c}\text { Índice de } \\
\text { Jaccard }\end{array}$ \\
\hline Caraigres (Café) & Escarabajos & 4 & 1 & 8 & 0,57 \\
& Mariposas & 19 & 33 & 23 & 0,25 \\
& Árboles & 9 & 8 & 10 & 0,29 \\
& Hierbas & 27 & 20 & 16 & 0,23 \\
Alfaro Ruíz (Hortalizas) & Escarabajos & 4 & 4 & 4 & 1,00 \\
& Mariposas & 11 & 6 & 10 & 0,37 \\
& Hierbas & 7 & 1 & 12 & 0,60 \\
Talamanca (Cacao-plátano) & Escarabajos & 5 & 0 & 11 & 0,40 \\
& Mariposas & 19 & 1 & 12 & 0,36 \\
& Árboles & 14 & 4 & 1 & 0,10 \\
& Hierbas & 12 & 5 & 5 & 0,12
\end{tabular}


CUADRO 4

Especies de mariposas únicas reportadas para cultivos orgánicos o convencionales en tres regiones de Costa Rica, $2009-2010$.

\begin{tabular}{|c|c|c|c|c|c|}
\hline \multicolumn{2}{|c|}{ Café } & \multicolumn{2}{|c|}{ Hortalizas } & \multirow{2}{*}{$\begin{array}{c}\text { Cacao-banano } \\
\text { Orgánico }\end{array}$} & \multirow{2}{*}{$\frac{\text { Plátano }}{\text { Convencional }}$} \\
\hline Orgánico & Convencional & Orgánico & Convencional & & \\
\hline $\begin{array}{l}\text { Megeuptychia } \\
\text { antonoe }\end{array}$ & Actinote anteas & Aphrissa statira & Danaus plexippus & $\begin{array}{l}\text { Adelpha cytherea } \\
\text { marcia }\end{array}$ & Hades noctula \\
\hline Megneuptychia libye & Aphrissa boisduvalii & $\begin{array}{l}\text { Cyanophrys } \\
\text { agricolor }\end{array}$ & Emesis tenedia & $\begin{array}{l}\text { Aeria eurimedia } \\
\text { agna }\end{array}$ & \\
\hline $\begin{array}{l}\text { Opsiphanes } \\
\text { tamarindi tamarindi }\end{array}$ & Appias drusilla & $\begin{array}{l}\text { Dimorphia zathoe } \\
\text { pallidula }\end{array}$ & Eueides lineada & $\begin{array}{l}\text { Anartia fatima } \\
\text { fatima }\end{array}$ & \\
\hline Rekoa palegon & Astrapte alardus & Greta annette & $\begin{array}{l}\text { Papilio polixenes } \\
\text { stabilis }\end{array}$ & $\begin{array}{l}\text { Anthanassa drusilla } \\
\text { lelex }\end{array}$ & \\
\hline Taygetis kerea & Calephelis schausi & Lieinix nemesis & $\begin{array}{l}\text { Pieriballia } \\
\text { mandela } \\
\text { noctipennis }\end{array}$ & Calospila cilissa & \\
\hline Taygetis salvini & Calephelis sodalis & Marpesia marcella & & $\begin{array}{l}\text { Chlosyne janais } \\
\text { janais }\end{array}$ & \\
\hline Tegosa anieta anieta & Calycopis isobeon & Melinaea ethra lilis & & $\begin{array}{l}\text { Cissia } \\
\text { pseudoconfusa }\end{array}$ & \\
\hline $\begin{array}{l}\text { Pareuptychia } \\
\text { metaleuca }\end{array}$ & Celaenorrhinus eligius & $\begin{array}{l}\text { Oenomaus } \\
\text { ortygnus }\end{array}$ & & $\begin{array}{l}\text { Eueides aliphera } \\
\text { gracilis }\end{array}$ & \\
\hline $\begin{array}{l}\text { Parides iphidamas } \\
\text { iphidamas }\end{array}$ & $\begin{array}{l}\text { Cyanophrys } \\
\text { herodotus }\end{array}$ & Pyrgus communis & & $\begin{array}{l}\text { Eurema albula } \\
\text { albula }\end{array}$ & \\
\hline Parides photinus & Epargyreus exadeus & $\begin{array}{l}\text { Siproeta epaphus } \\
\text { epaphus }\end{array}$ & & $\begin{array}{l}\text { Eurema } \\
\text { xanthochlora } \\
\text { xanthochlora }\end{array}$ & \\
\hline Phoebis philea philea & Cyllosis pephredo & $\begin{array}{l}\text { Tithorea tarricina } \\
\text { pinthias }\end{array}$ & & $\begin{array}{l}\text { Heliconius erato } \\
\text { petiverana }\end{array}$ & \\
\hline Pierella luna luna & $\begin{array}{l}\text { Danaus eresimus } \\
\text { montezuma }\end{array}$ & & & Ithomia patilla & \\
\hline Leptotes sp. & $\begin{array}{l}\text { Eurytides protesilaus } \\
\text { dariensis }\end{array}$ & & & $\begin{array}{l}\text { Janatella } \\
\text { leucodesma }\end{array}$ & \\
\hline $\begin{array}{l}\text { Battus polydamas } \\
\text { polydamas }\end{array}$ & $\begin{array}{l}\text { Hamadryas } \\
\text { amphinome } \\
\text { mexicana }\end{array}$ & & & $\begin{array}{l}\text { Morpho } \\
\text { granadensis } \\
\text { polybaptus }\end{array}$ & \\
\hline Chiodes catillus & Hemiargus hanno & & & $\begin{array}{l}\text { Panthiades } \\
\text { bathildis }\end{array}$ & \\
\hline $\begin{array}{l}\text { Chloreuptychia } \\
\text { arnaea }\end{array}$ & Leptotes cassius & & & $\begin{array}{l}\text { Pareuptychia } \\
\text { metaleuca }\end{array}$ & \\
\hline Anteos maerula & Malpesia alcibiades & & & Rekoa marius & \\
\hline Anthanassa ardys & Marpesia petreus & & & $\begin{array}{l}\text { Siproeta stelenes } \\
\text { biplagiata }\end{array}$ & \\
\hline $\begin{array}{l}\text { Anthanassa drusilla } \\
\text { lelex }\end{array}$ & $\begin{array}{l}\text { Mechanitis menapis } \\
\text { saturata }\end{array}$ & & & $\begin{array}{l}\text { Taygetis laches } \\
\text { laches }\end{array}$ & \\
\hline
\end{tabular}


son las que pueden aprovechar el poco recurso que estos ofrecen. Por ejemplo, solamente se observaron algunas especies alimentándose de los plátanos en descomposición, tales como Hermeuptychia harmonia, $H$. Hermes y Cissia confusa, especies muy abundantes en hábitats alterados (Tobar \& Muhammad, 2010), y en una ocasión se detectó un individuo de la especie Caligo telemonius que encuentra su planta hospedera (genero Musa). Estas especies también fueron de las más comunes en los cultivos de cacao-banano orgánico y se alimentaban de frutos en descomposición de cacao.

La mayor abundancia de mariposas y escarabajos en cultivos orgánicos de café y cacao-banano que convencionales, coincide con lo encontrado en Costa Rica y México para la comunidad de hormigas asociadas a cafetales, donde las abundancias disminuyen conforme aumenta la intensidad del manejo del cultivo (Perfecto \& Vandermeer, 1996; Armbrecht \& Perfecto, 2003). Así mismo, Hall (2003) reportó que la abundancia de escarabajos fue mayor en cultivos orgánicos de café comparado con café con poró, banano y eucalipto usados como sombra. Igualmente, Suatunce (2002) reportó para nuestra misma área de estudio en cultivos de cacao en Talamanca una relación positiva entre el número de especies de plantas presentes en estos cultivos con la riqueza de escarabajos. Para las especies de escarabajos coprófagos la cobertura arbórea es uno de los principales factores que influyen en la diversidad de este grupo (Lobo, Lumaret \& Jay-Robert, 1998).

Otro hecho que puede influir en la mayor abundancia de escarabajos en los cultivos orgánicos, es la presencia de animales domésticos como cerdos y perros. Las excretas de estos animales aumentan el recurso alimenticio para este grupo influyendo positivamente en el aumento de su población. Por otra parte, Suatance (2002) y Harvey, Gonzalez y Somarriba (2006) concluyen que los cacaotales de la región de Talamanca pueden albergar una diversidad de escarabajos coprófagos comparable a la de los bosques que los rodean. La detección de especies únicas de escarabajos (Eurysternus foedus y Uroxys platypyga), se asocia a la presencia de mamíferos en bosques (Peck \& Howden, 1984, Estrada, Halffter, Coates-Estrada \& Meritt, 1993), influyendo en las diferencias encontradas entre cultivos y bosque.

En lo que se refiere a la vegetación, las fincas orgánicas y convencionales de café no presentaron diferencias en la cantidad de especies de vegetación utilizada como sombra, pero sí presentaron diferencias importantes en composición. Por ejemplo en fincas orgánicas se registró especies remanentes del bosque original de la zona como Guanacaste, Cocobolo y Sangrillo, las cuales no se registraron en sistemas convencionales. Por otra parte, los cultivos de hortalizas orgánicos son más ricos en número de especies y abundancia de vegetación acompañante que los convencionales, sin embargo no parecen tener influencia en la abundancia o riqueza de mariposas o escarabajos en ése sitio. En los cultivos de hortalizas la temperatura del suelo podría tener un efecto negativo en estos grupos. Rivera \& Albrecht (2005) reportaron que a medida que aumenta la temperatura del sitio disminuye el número de especies de hormigas, por lo que este tipo de cultivo presenta mayor exposición del suelo que los cultivos de café y cacao-banano podría estar influyendo en encontrar menor abundancia de mariposas y escarabajos.

\section{Composición}

El grupo más similar entre cultivos y bosque fueron los escarabajos. En México, Estrada, Coates-Estrada, Anzures y Cammarano (1998) encontraron que el 77\% de las especies de escarabajos coprófagos registradas estuvieron presentes en hábitat diferentes al bosque como plantaciones de café, cacao y cítricos, evidenciando su plasticidad ecológica o capacidad de adaptarse a las condiciones que les ofrecen diferentes tipos de sistemas productivos. Por otro lado, Hernandez et al. (2003) reportó que varias especies como Dichotomius satanas y Onthophagus praecellens disminuyen su abundancia en época seca, esto pudo favorecer la detección de una menor cantidad de especies y por tanto la mayor similitud entre tipos de cultivo.

Para los grupos evaluados se encontró mayor cantidad de especies exclusivas a cultivos orgánicos que a convencionales con excepción de las mariposas, en las que se registró mayor número de especies exclusivas en cultivos de café convencional. Muchas de las especies de mariposas únicas para cultivos convencionales pertenecen a las familias Riodinidae, Lycaeniidae y Hesperiidae, estas especies pueden visitar una gran cantidad de hábitat, pero muchas prefieren áreas abiertas (DeVries 1987). Por otro lado, todos los cultivos orgánicos se encontraban cercanos a parches de bosque, esto pudo favorecer que muchas de las especies de sistemas orgánicos se compartieran con las registradas en el bosque (Horner-Devine et al. 2003).

La diferencia encontrada en composición de vegetación herbácea y arbórea entre sistemas orgánicos y convencionales está asociada al manejo que se le da al sistema productivo. Por ejemplo, la agricultura orgánica conlleva un incremento de la abundancia de las especies arvenses, por la eliminación de herbicidas pero también un cambio en la composición florística (Castro \& Amador 2007). Esta diferencia no fue tan marcada en el caso de vegetación para sombra de café, sin embargo en cultivos orgánicos se encontró especies maderables como el cocobolo (Dalbergia retusa), guanacaste (Enterolobium cyclocarpum) y 
laurel (Cordia alliodora) que no se registraron en cultivos convencionales.

Con este estudio, a pesar de la variedad de condiciones de las fincas estudiadas, evidenciamos como el manejo orgánico está contribuyendo a la conservación de diferentes grupos ecológicos, tanto en riqueza de especies, número de individuos y mayor complejidad en su composición. Cabe resaltar que estos resultados podrían cambiar si los remanentes de bosque dejan de estar presente alrededor de estos cultivos ya que ninguno de estos agroecosistema brindan los mismos nichos ni hábitats que brindan los parches de bosque original (Horner-Devine et al. 2003, Fuller et al. 2005, Pérez 2008). De ahí la importancia de promover la agricultura orgánica, pero además la conservación de fragmentos de bosque dentro o cercanos a las fincas.

\section{AGRADECIMIENTOS}

A los productores y familias de las comunidades de Aserrí, Acosta, Tapesco, Zarcero, Shiroles y La perla por su hospitalidad y amistad. Marco Otárola, Angel Solís y Germán Vega colaboraron en la identificación de los especímenes. Branko Hilje y dos revisores anónimos brindaron consejos para mejorar el manuscrito. Esta investigación fue financiada por la Corporación Educativa para el Desarrollo Costarricense (CEDECO).

\section{REFERENCIAS}

Armbrecht, I., \& Perfecto, I. (2003). Litter-dwelling ant species richness and predation potential within a forest fragment and neighboring coffee plantations of contrasting habitat quality in Mexico. Agriculture, Ecosystems and Environment, 97(1-3), 107-115.

Becker, B. (1995). Indicator plants for sustainability assessment of tropical production systems. Journal of Applied Botany, 68(3-4), 145-151.

Brown, K.S., \& Hutchings, R.W. (1997). Disturbance, fragmentation, and the dynamic of diversity in Amazonian forest butterflies. In W. F. Lawrence, \& Bierregaard, R. O. (Eds.). Tropical forest remnants: Ecology, management, and conservation of fragmented communities. Chicago: University of Chicago Press.

Castro, J., \& Amador, M. (2007). Proyecto emisión de gases de efecto invernadero y agricultura orgánica: sistematización de metodologías. San José, Costa Rica: Cedeco.

DeVries, P.H. (1987). The butterflies of Costa Rica and their natural history. Ithaca, New York: Cornell University Press.

Estrada, A., Halffter, G., Coates-Estrada, R., \& Meritt, D.A.Jr. (1993). Dung beetles attracted to mammalian herbivore (Alouatta palliata) and omnivore (Nasua narica) dung in the tropical rain forest of Los Tuxtlas, Mexico. Journal of Tropical Ecology, 9(2), 45-54.

Estrada A., Coates-Estrada, R., Anzures, A., \& Cammarano, P.L. (1998). Dung and carrion beetles in tropical rain forest fragments and agricultural habitats at Los Tuxtlas, Mexico. Journal of Tropical Ecology, 14(5), 577-593.

Feber, R.E., Firbank, L.G., Johnson, P.J., \& Macdonald, D.W. (1997). The effects of organic farming on pest and non pest butterfly abundance. Agriculture, Ecosystems and Environment, 64(2), 133-139

Finegan, B., Hayes, J., Delgado, D., \& Gretzinger, S. (2004). Monitoreo ecológico del manejo forestal en el trópico húmedo. Fondo Mundial para la Naturaleza, WWF, Centroamérica. San José, Costa Rica: CATIE

Florian, E. M. (2005). Tropical bird assemblages in coffee agroforestry systems: exploring the relationships between landscape context, structural complexity and bird communities in the Turrialba-Jiménez Biological Corridor (Tesis de maestría inédita). Centro Agronómico Tropical de Investigación y Enseñanza, Turrialba, Costa Rica.

Fuller, R.J., Norton, L.R, Feber, R.E., Johnson, P.J., Chamberlain, D.E., Joys, A.C, ... \& Firbank, A.C. (2005). Benefits of organic farming to biodiversity vary among taxa. Biology Letters, 1(2), 431-434.

Gallina, S., Mandujano, S., \& Gonzalez-Romero, A. (1996). Conservation of mammalian biodiversity in coffee plantations of Central Veracruz, Mexico. Agroforestry Systems, 33(1), 13-27.

George, A. (2006). Estudio comparativo de indicadores de calidad de suelo en fincas de café orgánico y convencional en Turrialba, Costa Rica. (Tesis de maestría inédita). Centro Agronómico Tropical de Investigación y Enseñanza, Turrialba, Costa Rica.

Guillen, C., Soto-Adames, F. \& Springer, M. (2006). Diversidad y abundancia de colémbolos edáficos en un bosque primario, un bosque secundario y un cafetal en Costa Rica. Agronomía Costarricense, 30(2), 7-17.

Guiracocha G., Harvey, C., Somarriba, E., Krauss, U. \& Carrillo, E. (2001). Conservación de la biodiversidad en sistemas agroforestales con cacao y banano en Talamanca, Costa Rica. Agroforesteria en las Américas, 8(30), 7-11.

Hald, A.B. (1999). Weed vegetation (wild flora) of long established organic versus conventional cereal fields in Denmark. Annals of Applied Biology, 134(3) 307-314.

Hall, S. (2003). Biodiversity conservation in agroecosystems: A comparison of surface-dwelling beetle diversity in various shade coffee production systems in Costa Rica., Toronto, Canada: FES Outstying Graduate Student Paper Series 7.

Hanski, I., \& Cambefort, Y. (1991). Resource partitioning, In I. Hanski, \& Cambefort, Y (Eds.), Dung beetle ecology. New Jersey: Princeton University Press.

Harvey, C.A., Gonzalez, J. \& Somarriba, E. (2006). Dung beetle and terrestrial mammal diversity in forests, indigenous agroforestry systems and plantain monocultures in 
Talamanca, Costa Rica. Biodiversity \& Conservation, 15(2), 555-585.

Hernández, B., Maes, J.M., Harvey, C.A., Vílchez, S., Medina, A., \& Sánchez, D. (2003). Abundancia y diversidad de escarabajos coprófagos y mariposas diurnas en un paisaje ganadero en el departamento de Rivas, Nicaragua. Agroforestería en las Américas, 10(39-40), 93-102.

Hole, D.G., Perkins, A.J., Wilson, J.D., Alexander, I.H., Grice, P.V., \& Evans, A.D. (2005). Does organic farming benefit biodiversity? Biological Conservation, 122(1), 113-130.

Horner-Devine, M.C., Daily, G.C., Ehrlich, P.R., \& Boggs, C.L. (2003). Countryside biogeography of tropical butterflies. Conservation Biology, 17(1), 168-177.

Jeanneret, P., Schüpbach, B., Pfiffner, L., \&Walter, T. (2003). Arthropod reaction to landscape and habitat features in agricultural landscapes. Landscape Ecology, 18(3), 253-263.

Klein, B.C. (1989). Effects of forest fragmentation on dung y carrion beetle communities in Central Amazonia. Ecology, 70(6), 1715-1725.

Lobo, J.M., Lumaret, J.P., \& Jay-Robert, P. (1998). Sampling dung beetles: effect of abiotic factors and farm practices. Pedobiologia, 42(3), 252-266.

Matson, P.A., Parton, W.J., Power, A.G., \& Swift, M.J. (1997). Agricultural intensification and ecosystem properties. Science, 277(5325), 504-509.

Moguel, P., \& Toledo, V.M.. (1999). Biodiversity conservation in traditional coffee systems of Mexico. Conservation Biology, 13(1), $11-21$.

Morón, M.A. (2004). Escarabajos. 200 millones de años de evolución. Zaragoza, España: Instituto de Ecología. A.C. y Sociedad Entomológica Aragonesa.

Peck, S.B., \& Howden, H.F. (1984). Response of a dung beetle guild to different sizes of dung bait in a Panamanian rainforest. Biotropica, 16(1):235-238.

Pérez, O. (2008). Evaluación de la biodiversidad de mariposas diurnas presentes en sistemas agroforestales modernos con café en el Corredor Biológico Volcánica Central-Talamanca, Costa Rica. (Tesis de maestría inédita) Centro Agronómico Tropical de Investigación y Enseñanza. Turrialba, Costa Rica.
Perfecto, I. \& Vandermeer, J. (1996). Microclimatic changes and the indirect loss of ant diversity in a tropical agroecosystem. Oecologia, 108(3), 577-582.

Perfecto, I., Mas, A., Dietsch, T., \& Vandermeer, J. (2003). Conservation of biodiversity in coffee agroecosystems: a tri-taxa comparison in southern Mexico. Biodiversity and Conservation, 12(6), 1239-1252.

Real, R., \& Vargas, J.M. (1996). The probabilistic basis of Jaccard's index of similarity. Systematic Biology, 45(3), 380-385.

Rivera, L. \& Armbrecht, I. (2005). Diversidad de tres gremios de hormigas en cafetales de sombra, de sol y bosques de Risaralda. Revista Colombiana de Entomologia, 31(1), 84-96.

Sans, F.X. (2007). La diversidad de los agroecosistemas. Ecosistemas, 16(1), 44-49.

Schroth, G., da Fonseca, B.A., Harvey, C.A., Gascon, C., Vasoncelos, H. L., Izac, R.A.M.N. \& Wilkie, D.S. 2004. Agroforestry and biodiversity conservation in tropical landscapes. In G. Schroth, Fonseca, G.A.B., Harvey, C.A., Gascon, C., Vasconcelos, H.L., \& Izac A.M.N. (Eds.), Agroforestry and biodiversity conservation in tropical landscapes. Washington: Island Press.

Suatunce, C.J.P. (2002). Diversidad de escarabajos estiercoleros en bosques y en cacaotales de diferente estructura y composición florística, Talamanca, Costa Rica. (Tesis de maestría inédita). Centro Agronómico Tropical de Investigación y Enseñanza, Turrialba, Costa Rica.

Swift, M.J., Vyerrneer, J., Rarnakrishnan, P.S., Yerson, J.M., Ong, C.K., \& Hawkinss, B.A. (1996). Biodiversity and agroecosystem function. In H.A. Mooney, Cushrnan, J.H., Medina, E., Sala, O.E., \& Schulze, E.D. (Eds.). Functional roles of biodiversity: a global perspective. New York: John Wiley and Sons Ltd.

Tobar, L.D.E. \& Muhammad, I. (2010). ¿Las cercas vivas ayudan a la conservación de la diversidad de mariposas en paisajes agropecuarios? Revista Biología Tropical, 58(1), 447-463.

Thomas, C. (1991). Habitat use and geographic range of butterflies from the wet lowlands of Costa Rica. Biological Conservation, 55(3), 269-281.

Tosi J.A. (1969). República de Costa Rica: mapa ecológico según la clasificación de zonas de vida de L.R. Holdridge. San José, Costa Rica: Centro Científico Tropical.

Vulinec, K. (2000). Dung beetles (Coleoptera: Scarabaeidae), monkeys, and conservation in Amazonia. Florida Entomologist, 83(3), 229-241. 
